\title{
Validation of Real-Time 3d Echocardiography Left Ventricular Volume- Time Curves with Cardiac MRI and Clinical Utilization of Emptying and Filling Rates
}

William L Duvall' ${ }^{1}$, Jeffrey Bander ${ }^{1}$, Lee I Korff-Korn ${ }^{1}$, Andrew Krasner ${ }^{2}$, Man Piu Wong ${ }^{3}$ Usman Baber $^{1}$, Javier Sanz ${ }^{1}$, Samantha Buckley ${ }^{1}$, Lori B Croft ${ }^{1}$ and Martin E Goldman ${ }^{1 *}$

${ }^{1}$ Mount Sinai Heart, Mount Sinai Medical Center, New York, USA

${ }^{2}$ Mount Sinai Department of Medicine, Mount Sinai Medical Center, New York, USA

${ }^{3}$ Mount Sinai School of Medicine, Mount Sinai Medical Center, New York, USA

\begin{abstract}
Background: 3D echocardiography derived volumetric data can be used to generate left ventricular volumetime curves and has the potential to elucidate aspects of contractility, systolic and diastolic function in normal and disease states. We sought to establish the validity and reproducibility of these volume-time curves and apply novel parameters derived from these curves to a clinical population.

Methods: Consecutive patients, who underwent cardiac MRI and echocardiography within 12 hours, were used to compare volume-time curves. Inter- and intra observer variability of the 3D echocardiography volume-time curves was assessed. Peak emptying rates, peak filling rates, and peak systolic acceleration were measured in a normal population (normal Ejection Fraction (EF) and no significant valvular disease) and their change with age was evaluated.

Results: 16 patients with an average EF of $55 \% \pm 10 \%$ underwent cardiac MRI and 3D echocardiography. There was no significant difference between the systolic and diastolic slopes of the volume-time curves derived by the two methods with good correlation for both systole $(r=0.62, p<0.0001)$ and diastole $(r=0.43, p=0.0025)$. In 50 normal patients aged 19-91, peak emptying rate, peak filling rate, and peak systolic acceleration all decreased with age.

Conclusion: 3D echocardiography volume-time curves correlate well with the gold standard of cardiac MRI. The novel 3D parameters of peak emptying rate, peak filling rate, and peak systolic acceleration may prove to be helpful in the assessment of systolic and diastolic function and provide insight into ventricular performance.
\end{abstract}

Keywords: Three dimensional transthoracic echocardiography; Systolic function; Diastolic function

Abbreviations: LV: Left Ventricle; EF: Ejection Fraction; 2D: Two Dimensional; 3D: Three Dimensional; CAD: Coronary Artery Disease; MRI: Magnetic Resonance Imaging; CT: Computed Tomography; EDV: End Diastolic Volume; ESV: End Systolic Volume; SV: Stroke Volume

\section{Introduction}

Echocardiographic assessment of Left Ventricular (LV) function, both systolic and diastolic, is important for the management of patients but is currently limited. Systolic function is derived by providing a global number (Ejection Fraction (EF)) based on single or bi-plane slices, and diastolic function is assessed by sampling small volumes (tissue Doppler) or single thin slices (2D strain). Real time transthoracic $3 \mathrm{D}$ echocardiography, may overcome $2 \mathrm{D}$ limitations by imaging the entire volume of the left ventricle and providing volumetric data rather than single plane or sample area data. $3 \mathrm{D}$ volumetric data has the potential to assess ventricular function using novel systolic and diastolic performance parameters. 3D datasets can be used to generate change in global LV volume over time curves which have the potential to elucidate aspects of contractility, systolic function, and diastolic function in normal and disease states. Analysis of these volume-time curves includes deriving: peak systolic emptying rates $(\mathrm{ml} / \mathrm{s})$, peak diastolic filling rates $(\mathrm{ml} / \mathrm{s})$, and peak systolic acceleration $\left(\mathrm{ml} / \mathrm{s}^{2}\right)$ (Figure 1).

Previous work on peak filling and emptying rates has used gated blood pool scans, cineangiography, invasive hemodynamics, and cardiac MRI [1-8]. These studies demonstrated a distinct separation in peak emptying between patients with normal and abnormal EF's and peak filling rates were abnormal in a high percentage of patients with Coronary Artery Disease (CAD), both with normal and abnormal EF's $[1,2]$. Peak emptying and filling rates correlated well with invasive hemodynamic measurements such as $\mathrm{EF},+\mathrm{dP} / \mathrm{dt},-\mathrm{dP} / \mathrm{dt}$, and LV end diastolic pressure [5]. Several small studies have also investigated using $3 \mathrm{D}$ echocardiography to generate volume-time curves along with peak emptying and filling rates [9-11]. 3D echocardiography has several advantages compared to gated blood pool scans, cineangiography, and cardiac MRI. It is non-invasive, portable, involves no radiation exposure, has short image acquisition time, better spatial resolution, and images the entire ventricular volume not counts or single/bi-plane slices.

The novel 3D systolic and diastolic performance parameters of peak filling and emptying rates have the potential to contribute to

*Corresponding author: Martin E Goldman, MD, Mount Sinai Medical Center, Box 1030, One Gustave L Levy Place, New York, NY 10029, USA, Tel: (212) 241-4040; Fax: (212) 426-6376; E-mail: martin.goldman@mountsinai.org

Received May 19, 2013; Accepted June 05, 2013; Published June 12, 2013

Citation: Duvall WL, Bander J, Korff-Korn LI, Krasner A, Wong MP, et al. (2013) Validation of Real-Time 3d Echocardiography Left Ventricular Volume-Time Curves with Cardiac MRI and Clinical Utilization of Emptying and Filling Rates. J Cardiovasc Dis Diagn 1: 112. doi:10.4172/2329-9517.1000112

Copyright: @ 2013 Duvall WL, et al. This is an open-access article distributed under the terms of the Creative Commons Attribution License, which permits unrestricted use, distribution, and reproduction in any medium, provided the original author and source are credited. 
Citation: Duvall WL, Bander J, Korff-Korn LI, Krasner A, Wong MP, et al. (2013) Validation of Real-Time 3d Echocardiography Left Ventricular VolumeTime Curves with Cardiac MRI and Clinical Utilization of Emptying and Filling Rates. J Cardiovasc Dis Diagn 1: 112. doi:10.4172/23299517.1000112

Page 2 of 7

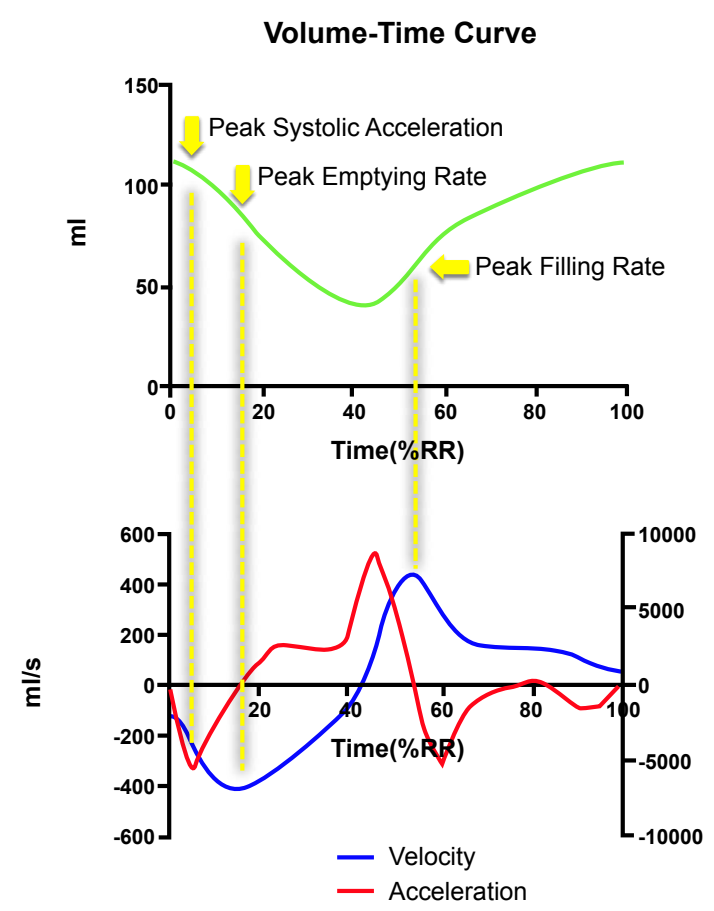

$\stackrel{3}{\stackrel{3}{N}}$

Figure 1: Volume-Time Curves and Derivation of Peak Emptying Rate, Peak Filling Rate, and Peak Systolic Acceleration.

the current assessment of left ventricular function in patients with subclinical disease or heart failure with normal EF's. The current diagnosis of diastolic dysfunction is based on Doppler derived diastolic filling parameters whose diagnostic accuracy was established as a result of these conventional echocardiographic parameters worsening with age [12-14]. Normal aging has been shown to result in reduced LV filling in early diastole and increased filling at atrial contraction and in most individuals the peak $\mathrm{E}$ and $\mathrm{A}$ wave velocities become approximately equal during the sixth and seventh decade of life [1518]. Studies of the general population have shown the prevalence of diastolic dysfunction by Doppler measurements to be as high as 28 $38 \%$ with the prevalence increasing with age $[14,19]$. These novel $3 \mathrm{D}$ performance parameters may be able to add to the assessment of diastolic function by augmenting the number of tools available to make the diagnosis.

We set out to establish 3D echocardiography derived volume-time curves as a valid measurement and a potential clinical tool. First, we sought to validate $3 \mathrm{D}$ echocardiography derived volume-time curves, and second we applied them to a clinical population. To establish the validity and reproducibility of this novel volumetric approach, we compared 3D echocardiography derived volume-time curves with those obtained from cardiac MRI. We also assessed the inter- and intraobserver variability of the $3 \mathrm{D}$ echocardiography volume-time curves and the derived peak emptying and filling rates. To clinically evaluate these novel 3D performance parameters as a potential diagnostic tool, we chose a normal population and assessed changes in the parameters with age.

\section{Methods}

\section{Study design}

In a study design approved by the Mount Sinai Medical Center's
Institutional Review Board, we studied two groups of subjects to both prospectively validate the $3 \mathrm{D}$ echocardiography derived volume time curves with a gold standard and retrospectively demonstrate possible clinical applications.

\section{Validation cohort}

We studied 16 consecutive patients over the age of 18 who consented to the study protocol with acceptable 3D echocardiography images who underwent a 1.5 Tesla (Magnetom Sonata', Siemens Medical Solutions, Erlangen, Germany) or a 3.0 Tesla (Achieva, Philips Medical Systems, Best, The Netherlands) cardiac MRI followed by a 3D echocardiogram (Philips IE-33, Andover, MA) within 12 hours.

\section{Clinical cohort}

The first 50 patients with adequate acoustic windows presenting for a clinically indicated transthoracic echocardiogram over a three month period from June 2008 through August 2008 who were imaged on the Philips IE-33 platform were included for analysis. Patients with an $\mathrm{EF}$ of $<45 \%$, significant (moderate or greater) valve disease, and atrial fibrillation were excluded. Patients were divided into four age groups $(<40,41-59,61-79$, and $>80$ years old $)$ for analysis.

\section{D Image acquisition and processing}

A standard transthoracic echocardiogram was performed and then high-resolution real time transthoracic 3D echocardiography images in the apical view were acquired with an X3-1 Philips transthoracic probe during a breath hold. 3D processing and volume-time curves were generated with commercially available software (Philips Q-Lab 5.0). From the volume-time curves exported from Philips Q-Lab, peak emptying rates $(\mathrm{ml} / \mathrm{s})$, peak filling rates $(\mathrm{ml} / \mathrm{s})$, and peak systolic acceleration $\left(\mathrm{ml} / \mathrm{s}^{2}\right)$ were generated using custom written software. Left ventricular EF, presence and severity of valvular disease, and common measures of diastolic function including trans-mitral pulse wave $\mathrm{E} / \mathrm{A}$, tissue Doppler e'/a' at the mitral annulus, E/e' ratio were recorded from the 2D images. Left ventricular 3D EF, End Diastolic Volumes (EDV), End Systolic Volumes (ESV), and Stroke Volumes (SV) were recorded from the standard $3 \mathrm{D}$ processing.

\section{Cardiac MRI image acquisition and processing}

Patients in the validation cohort were imaged in the supine position using dedicated multi-element surface coils and retrospective electrocardiographic gating. Multiple short-axis cine views covering the left ventricle from base to apex were acquired over 5-7 minutes with a standard segmented steady-state free precession sequence during limited end-expiratory breath-holds. Typical imaging parameters included repetition time $3.2 \mathrm{~ms}$, echo time $1.6 \mathrm{~ms}$, maximized flip angle, field of view adjusted to patient body size, matrix $256 \times 125$, lines per segment 11-15 (resulting in a temporal resolution of $35-50 \mathrm{~ms}$ ), reconstructed cardiac phases 25-30; receiver bandwidth $930 \mathrm{~Hz} /$ pixel). Images were then analyzed with dedicated software (Argus, Siemens Medical Solutions, Erlangen, Germany; or Extended MR Workspace Philips Medical Systems, Best, Netherlands). Left ventricular endocardial contours were traced from base to apex throughout the cardiac cycle. For each phase, LV volumes were obtained by Simpson's method and combined to generate volume-time curves.

\section{D echocardiography inter- and intra-observer variability}

For inter-observer variability assessment, two level 3 echocardiographers (WLD and MEG) independently and blindly processed the $3 \mathrm{D}$ datasets from the 16 patients in the validation cohort 
Citation: Duvall WL, Bander J, Korff-Korn LI, Krasner A, Wong MP, et al. (2013) Validation of Real-Time 3d Echocardiography Left Ventricular VolumeTime Curves with Cardiac MRI and Clinical Utilization of Emptying and Filling Rates. J Cardiovasc Dis Diagn 1: 112. doi:10.4172/23299517.1000112

Page 3 of 7

to generate volume-time curves. From the volume-time curves peak emptying rates and peak filling rates were generated using custom written software. For intraobserver variability assessment, two weeks after the initial 3D processing one of the echocardiographers, blindly and in random order, re-processed the 3D datasets. These volume-time curves were again used to generate peak emptying rates and peak filling rates.

\section{Statistical analysis}

The volume-time curves derived from cardiac MRI and 3D echocardiography were compared after dividing the curves into six segments, three each for both systole and diastole. Comparisons between MRI and 3D echocardiography-derived slope segments were made using paired t-tests and Pearson correlation coefficients, respectively. Inter- and intra-observer variability for peak emptying and peak filling rates was determined using Pearson's correlation coefficient.

Novel performance parameters of peak emptying rates, peak filling rates, and peak systolic acceleration in the normal clinical cohort were analyzed by age, E/e' Ratio, EF, EDV, and SV. Continuous variable were analyzed using a two-tailed paired Student's t-test and categorical variables using Chi-squared analysis. Independent associations between variables were assessed by linear regression.

\section{Results}

\section{Validation with cardiac MRI}

The 16 patients had a mean age of $50.7 \pm 13.1$ years, $56 \%$ were male, and the average EF was $54.9 \% \pm 10.3 \%$ (Table 1). There was no significant difference between the systolic and diastolic slopes of the volume-time curves derived using 3D echocardiography and cardiac MRI (Figure 2). There was good correlation between the two methods for both systole $(\mathrm{r}-0.62, \mathrm{p}<0.0001)$ and diastole $(\mathrm{r}-0.43, \mathrm{p}-0.0025)$.

\section{Inter- and intra-observer variability}

Inter-observer correlation for 3D echocardiography derived slopes was excellent: $\mathrm{r}-0.8$ for systole, $\mathrm{r}-0.98$ for diastole, $\mathrm{p}<0.0001$. Interobserver variability on the 16 patients in the validation cohort was low for both the peak emptying rate and the peak filling rate $(\mathrm{r}-0.88$ and $\mathrm{r}-0.93$ respectively, $\mathrm{p}<0.0001$ ) (Figure 3 ). Intraobserver variability on these patients was also good with $\mathrm{r}-0.71$ for peak emptying rate ( $\mathrm{p}$ $0.0019)$ and $\mathrm{r}-0.76$ for peak filling rate ( $\mathrm{p}-0.0006)$ (Figure 4$)$.

\section{Clinical cohort}

We studied 50 normal clinical patients for age related cardiac changes who had a mean age of $58.6 \pm 18.6$ years, $54 \%$ of whom were male (Table 1). The average left ventricular EF was $61.1 \% \pm 7.5 \%$.

Despite having a normal left ventricular EF, peak emptying rates,

\begin{tabular}{|c|c|c|}
\hline & Validation Cohort N = 16 & Clinical Cohort N =50 \\
\hline Age (yrs) & $50.7 \pm 13.1$ & $58.6 \pm 18.6$ \\
\hline Gender & & \\
\hline Male & $9(56 \%)$ & $27(54 \%)$ \\
\hline Female & $7(44 \%)$ & $23(46 \%)$ \\
\hline 3D Ejection Fraction $(\%)$ & $54.9 \% \pm 10.3 \%$ & $61.1 \% \pm 7.5 \%$ \\
\hline End Diastolic Volume $(\mathrm{ml})$ & $110.7 \pm 26.2$ & $89.4 \pm 30.9$ \\
\hline End Systolic Volume $(\mathrm{ml})$ & $50.7 \pm 19.0$ & $34.1 \pm 13.9$ \\
\hline Stroke Volume $(\mathrm{ml})$ & $60.0 \pm 15.6$ & $55.3 \pm 20.5$ \\
\hline
\end{tabular}

Table 1: Patient Characteristics.
A.

\section{Correlation of Systolic and Diastolic Slopes}

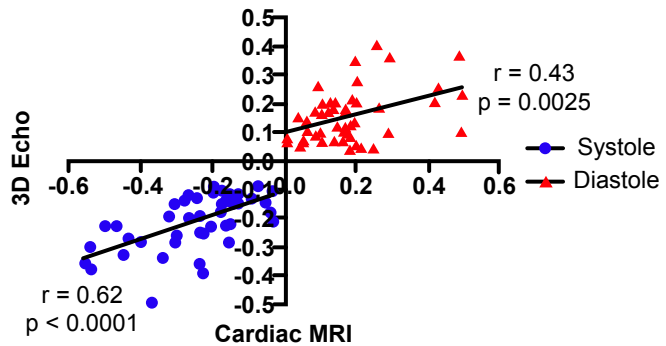

Comparison of slopes Derived by Cardiac MRI and 3D Echo

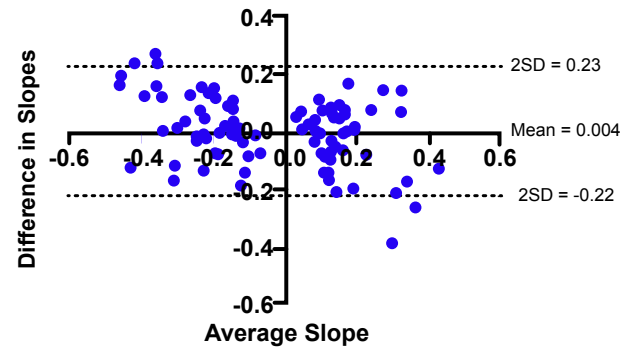

\section{D Echo \& Cardiac MRI Time Volume Curves}

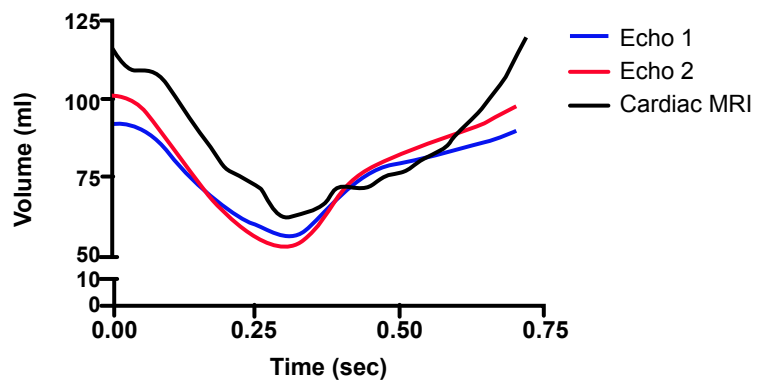

Figure 2: Correlation of systolic and diastolic slopes of time-volume curves between cardiac MRI and 3D echo (A), and example of single patient's time volume curves from two 3D echo readers and cardiac MRI (B).

systolic acceleration, and peak filling rates all decreased with age. When these patients were divided based on age range, compared to patients in the oldest group, patients in the youngest group had significantly faster peak emptying rate $(\mathrm{p}-0.02)$, peak filling rate $(\mathrm{p}$ $0.02)$, and peak systolic acceleration ( $\mathrm{p}-0.04)$ (Figure 5). When taken as a continuous variable, peak emptying rates $(\mathrm{p}-0.0007)$ and peak filling rates ( $\mathrm{p}-0.0031)$ both significantly decreased with age and peak systolic acceleration ( $\mathrm{p}-0.059)$ showed a trend towards slowing with age (Figure 6).

As potential novel measures of diastolic function, we compared them to common echocardiographic Doppler measures of diastolic function. All three novel parameters derived from 3D echocardiography had as strong or stronger association with age than E/e' ratio did (Figure 6 and 7). Peak diastolic filling rates poorly correlated with the $\mathrm{E} / \mathrm{e}^{\prime}$ ratio $(\mathrm{r}-\mathrm{-0.35}, \mathrm{p}-0.019)$ (Figure 7).

The novel 3D parameters of peak emptying rate, peak filling rate, and peak systolic acceleration were assessed for their association to EF and ventricular volumes such as EDV and SV (Table 2). While univariate analysis showed significant correlation between the $3 \mathrm{D}$ parameters and 
A.

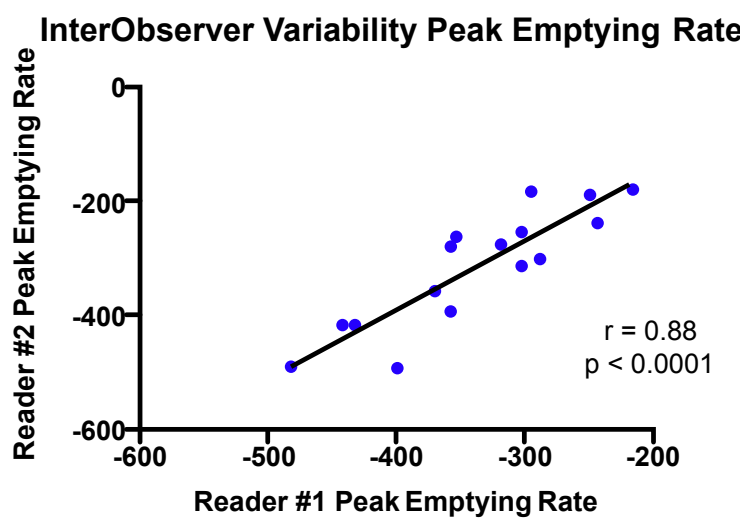

B.

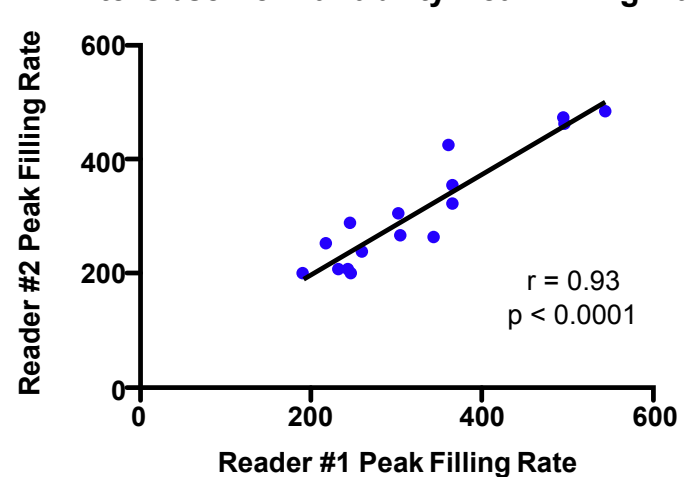

Figure 3: Interobserver Variability for (A) Peak Emptying Rate and (B) Peak Filling Rate.

the left ventricular variables, the association lost statistical significance in a multivariate model (consisting of age, EF, EDV, SV, and E/e' ratio) for all variables except peak emptying rate and stroke volume.

\section{Discussion}

This work demonstrated that real time 3D echocardiography generated volume-time curves correlated well with cardiac MRI generated curves and were not significantly different. The 3D echocardiography generated curves also had low inter-observer variability of measurements of derived slopes. The reproducibility of these novel parameters was good with minimal inter- and intraobserver variability for peak emptying rates and peak filling rates. For the most part, the novel 3D parameters provided information beyond of traditional measures of left ventricular function and volume based on multivariate analysis. These novel parameters were associated (weakly) with EF but had a stronger association with LV volumes (EDV and ESV). However in multivariate analysis, most associations to EF and volumes were lost.

In a small clinical cohort we also demonstrated that despite preserved LVEF, aging hearts manifested progressive changes in these novel systolic and diastolic performance variables. All three novel parameters, peak emptying rate, peak filling rate, and peak systolic acceleration, declined with age and had a stronger association with age than E/e' ratio. These novel systolic parameters worsen with age despite patients having normal left ventricular ejection fraction suggesting that the ventricular aging process involves both systole and diastole. This study demonstrates that $3 \mathrm{D}$ echocardiography is a feasible way to measure these novel parameters.

The parameters we derived from 3D echocardiography volume time curves have been derived from other modalities in the past. The initial work on left ventricular emptying and filling rates dates back to the 1970's and studied 113 patients and 29 normal subjects using single-plane cineangiograms $[3,4]$. Peak emptying rate correlated well with peak aortic flow rates and was not closely related to EF but to LV volume (EDV and SV) [3]. Peak diastolic filling rate was abnormal in patients with coronary disease and chronic valvular disease but was also related to volume characteristics of the LV (EDV \& SV) [4] Subsequent work with first pass radionuclide angiocardiograms in 22 patients without valvular regurgitation found that peak emptying rates correlated well with EF by angiography and allowed complete separation of normal and abnormal EF patients [1]. In addition, peak emptying rates increased eight-fold more than $\mathrm{EF}$ after isoproterenol infusion. In the largest study of 231 patients, radionuclide angiography derived peak filling rates were abnormal in a high percentage of patients at rest with coronary artery disease, both with normal and abnormal EF's [2] And in a study of 28 patients using gated radionuclide angiography and intracardiac pressure recordings peak ejection and filling rates correlated well with invasive hemodynamic measurements $(\mathrm{EF},+\mathrm{dP} /$ $\mathrm{dT},-\mathrm{dP} / \mathrm{dT}$, and LV end diastolic pressure) [5].

Older 3D echocardiography platforms have been used to generate

A.

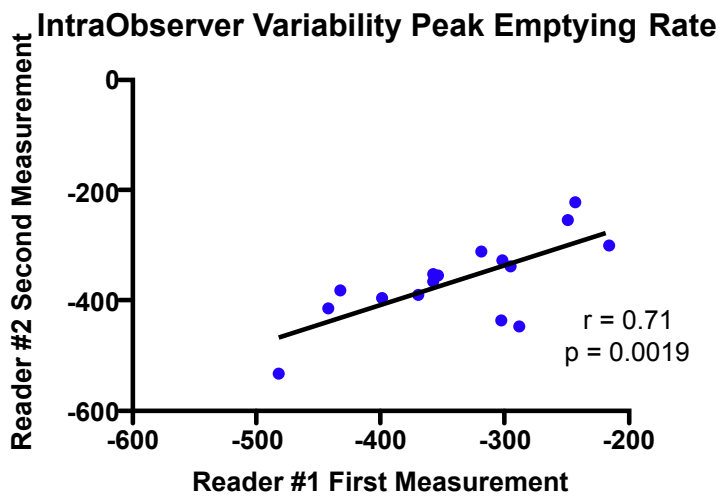

B.

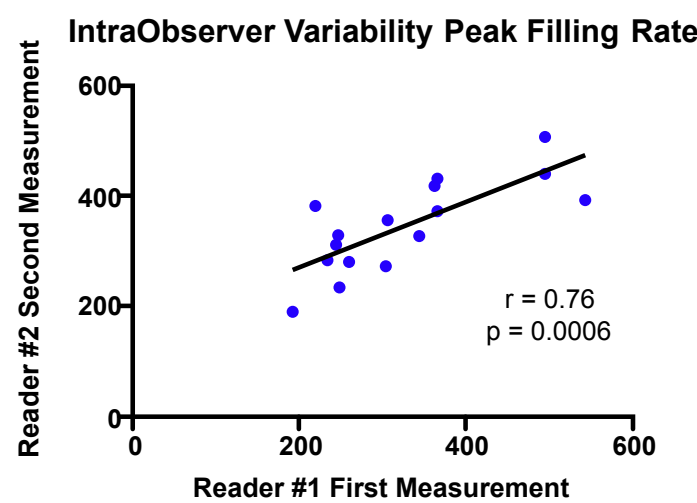

Figure 4: Intraobserver Variability for (A) Peak Emptying Rate and (B) Peak Filling Rate. 


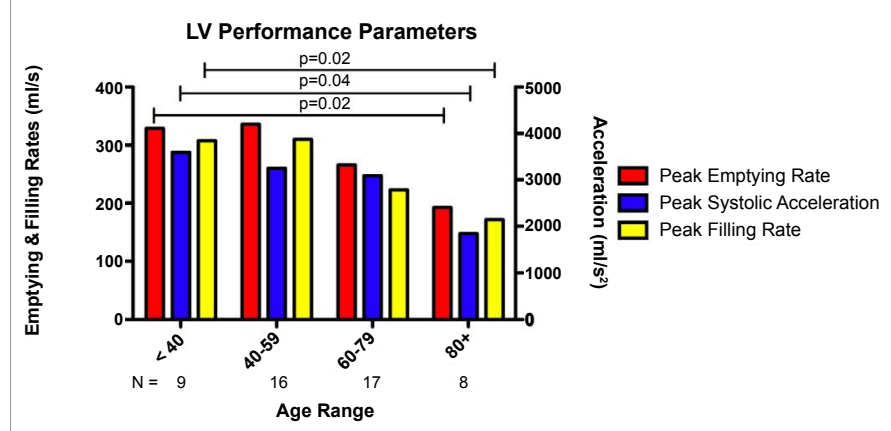

Figure 5: Changes in novel parameters with age dividing patients into four age groups.

A.

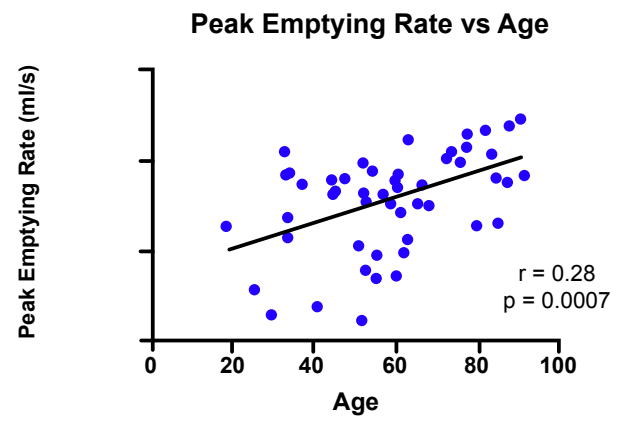

B.

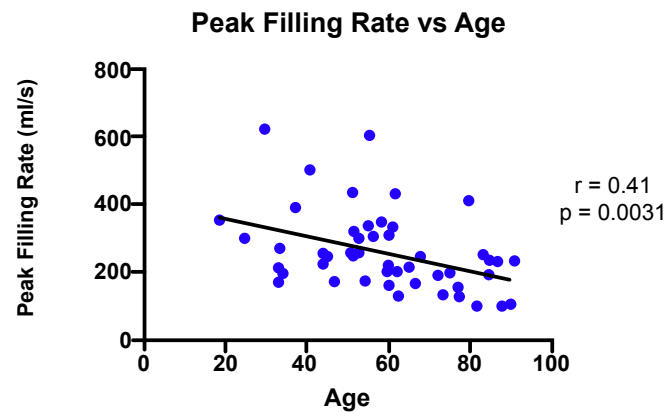

C.

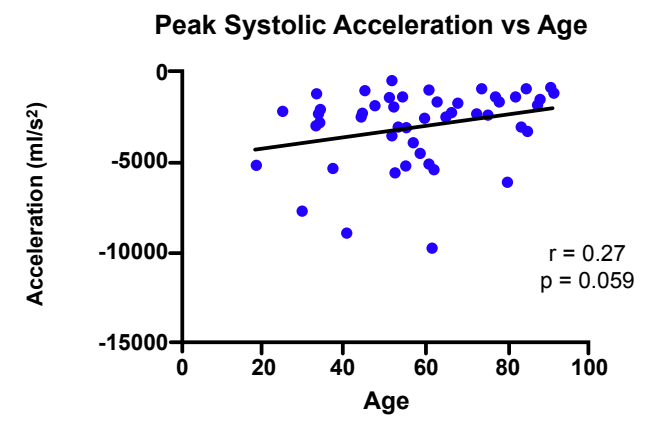

Figure 6: Novel 3D Parameters Versus Age. (A) Peak Emptying Rate, (B) Peak Filling Rate, and (C) Peak Systolic Acceleration.

volume-time curves and peak emptying and filling rates calculated [9-11]. While these echocardiography systems are no longer, or never were commercially available, they also showed good correlation with cardiac MRI. Differences were found in peak filling rates and peak emptying rates between small groups of patients without cardiovascular disease and those with heart failure, hypertension, and coronary artery disease $[10,11]$. Our study supports $3 \mathrm{D}$ echocardiography's ability to noninvasively assess left ventricular emptying and filling rates and finds differences in these parameters with increasing age in place of based on normal patient's age. 3D echocardiography has advantages over other imaging techniques including portability, improved spatial resolution (gated blood pool scans and myocardial perfusion imaging), fast image acquisition and analysis time (cardiac MRI), lack of ionizing radiation (nuclear cardiology and cardiac CT), and independence from potentially nephrotoxic agents (cardiac CT).

The rate of peak systolic emptying, the systolic ejection phase derived parameter may be affected by intrinsic factors (aging, ischemia, myocardial disease) and extrinsic factors (loading conditions, valve disease). In our group of otherwise normal subjects, ventricular

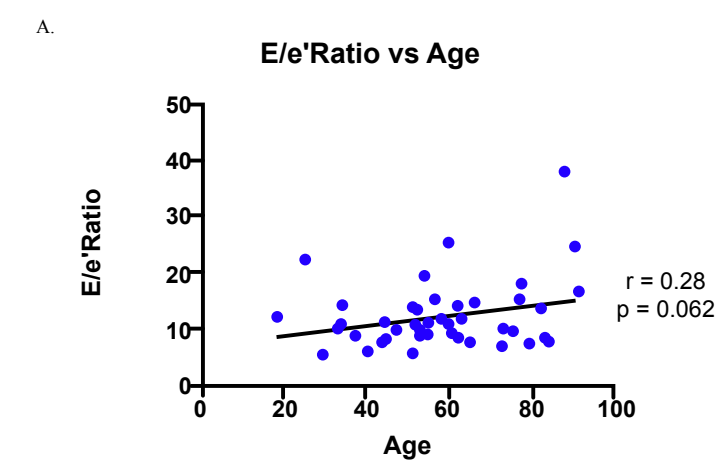

B.
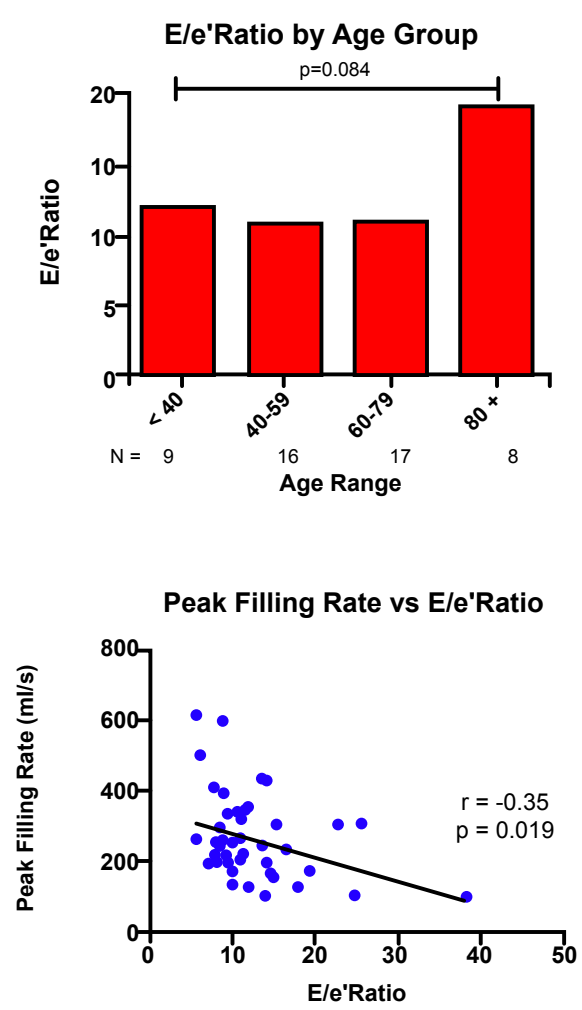

Figure 7: Correlation of E/e' Ratio with Age (A) and Difference in E/e' Ration by Age Group (B). The Correlation of Peak Filling Rate to E/e' Ratio (C). 
Citation: Duvall WL, Bander J, Korff-Korn LI, Krasner A, Wong MP, et al. (2013) Validation of Real-Time 3d Echocardiography Left Ventricular VolumeTime Curves with Cardiac MRI and Clinical Utilization of Emptying and Filling Rates. J Cardiovasc Dis Diagn 1: 112. doi:10.4172/23299517.1000112

Page 6 of 7

\begin{tabular}{|c|c|c|}
\multicolumn{4}{|c}{ Peak Emptying Rate } \\
\hline Characteristic & Univariate P value & Multivariate P Value \\
\hline Age & 0.0007 & 0.044 \\
\hline EF & 0.044 & 0.64 \\
\hline EDV & $<0.0001$ & 0.76 \\
\hline SV & $<0.0001$ & 0.027 \\
\hline E/e' Ratio & 0.093 & - \\
\hline \multicolumn{3}{|c|}{ Peak Filling Rate } \\
\hline Characteristic & Univariate P value & Multivariate P Value \\
\hline Age & 0.0031 & 0.76 \\
\hline EF & 0.042 & 0.23 \\
\hline EDV & $<0.0001$ & 0.31 \\
\hline SV & $<0.0001$ & 0.83 \\
\hline E/e' Ratio & 0.019 & 0.21 \\
\hline & Peak Systolic Acceleration \\
\hline Characteristic & Univariate P value & Multivariate P Value \\
\hline Age & 0.059 & 0.81 \\
\hline EF & 0.123 & - \\
\hline EDV & $<0.0001$ & 0.93 \\
\hline SV & $<0.0001$ & 0.14 \\
\hline E/e' Ratio & 0.036 & 0.20 \\
\hline
\end{tabular}

Table 2: Univariate and Multivariate Analysis of the Association of Novel 3D Parameters to Age, E/e' Ratio, EF, and Ventricular Volumes.

systolic performance as determined by peak emptying rates was slower in the older patients compared to younger subjects. This was in addition to a reduction in peak diastolic filling rates. The abnormalities in systolic ventricular performance were present despite a normal ejection fraction. While patients with diastolic heart failure virtually always exhibit normal indices of LV systolic performance, function, and contractility previous echocardiography Doppler studies have suggested that diastolic heart failure may be caused by subtle systolic dysfunction or exhibits a coexistence with systolic dysfunction [20-23]. In this current study, a subtle but measureable decline in ventricular systolic function as well as diastolic function was present in the aging heart and these novel parameters may in the future be able to aid in the diagnosis of normal EF heart failure.

\section{Limitations}

This study is limited by its small sample size and single center experience. 3D echocardiography is currently limited by image quality and the inability to acquire diagnostic images in a majority of patients. New technology may improve these shortcomings. The uni- and multivariate analysis may have been limited due to the narrow range of EF's ( $>45 \%$ ) since we were evaluating a group who by definition had normal LVEF.

\section{Conclusions}

These novel real time 3D echocardiography volume time curve derived parameters may, with further study, prove to be helpful in the assessment of systolic and diastolic function. They correlate well with the gold standard of cardiac MRI and yet are quicker and easier to obtain. Novel systolic and diastolic parameters derived from volumetric data may provide insight into ventricular performance not available by other imaging modalities. Further work in various disease states might demonstrate that these parameters could provide a more detailed noninvasive assessment of systolic function, may prove to be early markers of latent disease, and might simplify the evaluation of diastolic dysfunction.

\section{References}

1. Marshall RC, Berger HJ, Costin JC, Freedman GS, Wolberg J, et al. (1977) Assessment of cardiac performance with quantitative radionuclide angiocardiography: sequential left ventricular ejection fraction, normalized left ventricular ejection rate, and regional wall motion. Circulation 56: 820-829.

2. Bonow RO, Bacharach SL, Green MV, Kent KM, Rosing DR, et al. (1981) Impaired left ventricular diastolic filling in patients with coronary artery disease: assessment with radionuclide angiography. Circulation 64: 315-323.

3. Hammermeister KE, Brooks RC, Warbasse JR (1974) The rate of change of left ventricular volume in man. I. Validation and peak systolic ejection rate in health and disease. Circulation 49: 729-738.

4. Hammermeister KE, Warbasse JR (1974) The rate of change of left ventricular volume in man. II. Diastolic events in health and disease. Circulation 49: 739747

5. Magorien DJ, Shaffer P, Bush C, Magorien RD, Kolibash AJ, et al. (1984) Hemodynamic correlates for timing intervals, ejection rate and filling rate derived from the radionuclide angiographic volume curve. Am J Cardiol 53 567-571.

6. Hoff FL, Turner DA, Wang JZ, Barron JT, Chutuape MD, et al. (1994) Semiautomatic evaluation of left ventricular diastolic function with cine magnetic resonance imaging. Acad Radiol 1: 237-242.

7. Kawaji K, Codella NC, Prince MR, Chu CW, Shakoor A, et al. (2009) Automated segmentation of routine clinical cardiac magnetic resonance imaging for assessment of left ventricular diastolic dysfunction. Circ Cardiovasc Imaging 2: $476-484$.

8. Mendoza DD, Codella NC, Wang Y, Prince MR, Sethi S, et al. (2010) Impact of diastolic dysfunction severity on global left ventricular volumetric filling - assessment by automated segmentation of routine cine cardiovascular magnetic resonance. J Cardiovasc Magn Reson 12: 46.

9. Poutanen T, Ikonen A, Jokinen E, Vainio P, Tikanoja T (2001) Transthoracic three-dimensional echocardiography is as good as magnetic resonance imaging in measuring dynamic changes in left ventricular volume during the heart cycle in children. Eur J Echocardiogr 2: 31-39.

10. Zeidan Z, Erbel R, Barkhausen J, Hunold P, Bartel T, et al. (2003) Analysis of global systolic and diastolic left ventricular performance using volumetime curves by real-time three-dimensional echocardiography. J Am Soc Echocardiogr 16: 29-37.

11. Corsi C, Lang RM, Veronesi F, Weinert L, Caiani EG, et al. (2005) Volumetric quantification of global and regional left ventricular function from real-time three-dimensional echocardiographic images. Circulation 112: 1161-1170.

12. Paulus WJ, Tschöpe C, Sanderson JE, Rusconi C, Flachskampf FA, et al (2007) How to diagnose diastolic heart failure: a consensus statement on the diagnosis of heart failure with normal left ventricular ejection fraction by the Heart Failure and Echocardiography Associations of the European Society of Cardiology. Eur Heart J 28: 2539-2550.

13. Nagueh SF, Appleton CP, Gillebert TC, Marino PN, Oh JK, et al. (2009) Recommendations for the evaluation of left ventricular diastolic function by echocardiography. Eur J Echocardiogr 10: 165-193.

14. Yamada H, Goh PP, Sun JP, Odabashian J, Garcia MJ, et al. (2002) Prevalence of left ventricular diastolic dysfunction by Doppler echocardiography: clinical application of the Canadian consensus guidelines. J Am Soc Echocardiogr 15 1238-1244.

15. Gardin JM, Drayer JI, Weber M, Rohan MK, Knoll M, et al. (1987) Dopple echocardiographic assessment of left ventricular systolic and diastolic function in mild hypertension. Hypertension 9: II90-96.

16. Klein AL, Burstow DJ, Tajik AJ, Zachariah PK, Bailey KR, et al. (1994) Effects of age on left ventricular dimensions and filling dynamics in 117 normal persons. Mayo Clin Proc 69: 212-224.

17. Miyatake K, Okamoto M, Kinoshita N, Owa M, Nakasone I, et al. (1984) Augmentation of atrial contribution to left ventricular inflow with aging as assessed by intracardiac Doppler flowmetry. Am J Cardiol 53: 586-589.

18. Appleton CP (2008) Evaluation of diastolic function by two-dimensional and Doppler assessment of left ventricular filling including pulmonary venous flow. In Klein AL, Garcia MJ, (eds): Diastology. Philadelphia: Saunders Elsevier.

19. Kuznetsova T, Herbots L, López B, Jin Y, Richart T, et al. (2009) Prevalence 
Citation: Duvall WL, Bander J, Korff-Korn LI, Krasner A, Wong MP, et al. (2013) Validation of Real-Time 3d Echocardiography Left Ventricular VolumeTime Curves with Cardiac MRI and Clinical Utilization of Emptying and Filling Rates. J Cardiovasc Dis Diagn 1: 112. doi:10.4172/23299517.1000112

Page 7 of 7

of left ventricular diastolic dysfunction in a general population. Circ Heart Fail 2: 105-112.

20. Baicu CF, Zile MR, Aurigemma GP, Gaasch WH (2005) Left ventricular systolic performance, function, and contractility in patients with diastolic heart failure. Circulation 111: 2306-2312.

21. Yip G, Wang M, Zhang Y, Fung JW, Ho PY, et al. (2002) Left ventricular long axis function in diastolic heart failure is reduced in both diastole and systole: time for a redefinition? Heart 87: 121-125.
22. Petrie MC, Caruana L, Berry C, McMurray JJ (2002) "Diastolic heart failure" or heart failure caused by subtle left ventricular systolic dysfunction? Heart 87 : 29-31.

23. Yu CM, Lin H, Yang H, Kong SL, Zhang Q, et al. (2002) Progression of systolic abnormalities in patients with "isolated" diastolic heart failure and diastolic dysfunction. Circulation 105: 1195-1201. 